\title{
Evaluation of dosimetry characteristics of a Silicon Photo-Multiplier with a plastic scintillator by Cs-137
}

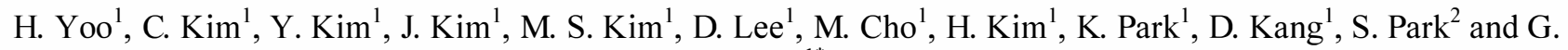 \\ $\mathrm{Cho}^{1 *}$
}

\begin{abstract}
A Silicon Photo Multiplier (SiPM) is states of arts radiation detector, it is alternative to PM-Tube. But, availability research of SiPM is not entirely satisfactory. In this study, we suggest the radiation detector module coupled with the SiPM and the plastic scintillator, and then, evaluate the availability of detector module for Cs-137 dosimetry.

To confirm the dosimetry characteristics, we experimented count difference depends on equivalent dose rate and equivalent dose comparing with MCNP calculation, and, calculating the conversion coefficient depends on count rate and total count that finding the region keeps the linearity of calculating the dose.

These experiment results means the SiPM with the plastic scintillator is able to use the dosimetry application for Cs-137. New detector module has the advantages that smaller volume than GM Tube and ion chamber and the lower price than indirect detector module using inorganic scintillator as CsI(TI). So, it will helpful to relieving the public anxiety about radiation exposure by popularizing the dosimetry device.
\end{abstract}

\section{INTRODUCTION}

A Silicon Photo-Multiplier (SiPM), thousands array of

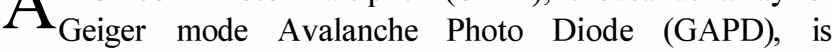
alternative radiation detector to PM-Tube. But, availability research is not entirely satisfactory [1-2]. A p-n junction in SiPM is optimized to indirect radiation detection, so, it needs a scintillation crystal. A plastic scintillator, made from polystyrene or anthracene, has very fast decay time smaller than $10 \mathrm{~ns}$ and simple manufacturing process to any shape or bigger size than inorganic scintillator. The density of plastic is so low about 1 , so the dominant radiation interaction with low energy gamma ray $(\sim 2 \mathrm{MeV})$ is Compton scattering. Energy attenuation coefficient of a plastic scintillator is similar with human tissue because of low density about 1. A plastic scintillator is mainly used in neutron spectroscopy and dosimetry of high energy space particle. But, this paper used a

Manuscript received November 15, 2013

This work was supported by the Center for Integrated Smart Sensors funded by the Ministry of Education, Science and Technology as Global Frontier Project (CISS-2012054201)

H. Yoo(telephone: 042-350-3861, e-mail: cmos@kaist.ac.kr), C. Kim, Y Kim, J. Kim, M. S. Kim, D. Lee, M. Cho, H. Kim, K. Park, D. Kang and G. Cho are with the Korea Advanced Institute of Science and Technology, Daejeon, S. Korea

S. Park is with Asan Medical Center, Seoul, S. Korea (telephone: 16887575, e-mail: Michael@amc.seoul.kr).
SiPM coupled with a plastic scintillator to confirm the availability for new application of Cs-137 dosimetry [3-7].

\section{MAterials AND MethodS}

\section{A. $\operatorname{SiPM}$}

The SiPM used in this study was SPM array 4 developed in Sensl, Ireland. SPM array 4 is 4 by 4 array of SiPMs for 16 channels and each SiPM consists of 3640 GAPDs in $3.16 \times$ $3.16 \mathrm{~mm}^{2}$ area. Among the 16 channel SiPMs, all of experiment setup in this study selected only 9 channels of SiPMs. So, each SiPM shared the anode and cathode parallel for operating in one large sensing area. The area of 9 channel SiPMs was $9.91 \times 9.91 \mathrm{~mm}^{2}$ and operating voltage was $30 \mathrm{~V}$, $+2.5 \mathrm{~V}$ above breakdown voltage. The figure of SPM array 4 is in Fig. 1.

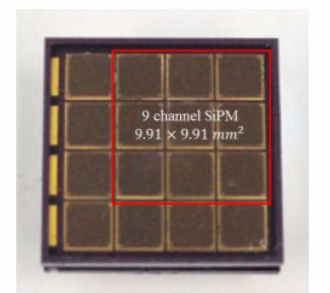

Fig. 1.9 channel area of SiPMs in SPM array 4

\section{B. Plastic scintillator}

The plastic scintillator which was coupled with SiPM was manufactured in Korea Advanced Institute on Science and Technology [3]. The shape of the plastic scintillator is $9 \mathrm{~mm}$ cube for isotropic detection regardless of entering radiation. The designed wavelength of output photon of plastic scintillator was $455 \mathrm{~nm}$ by mixing with the wavelength shifter as PPO and POPOP. The light emission spectrum of plastic scintillator is in Fig.2. And the manufactured plastic scintillator and the detector module coupled with the plastic scintillator and the SiPM are in Fig. 3. 


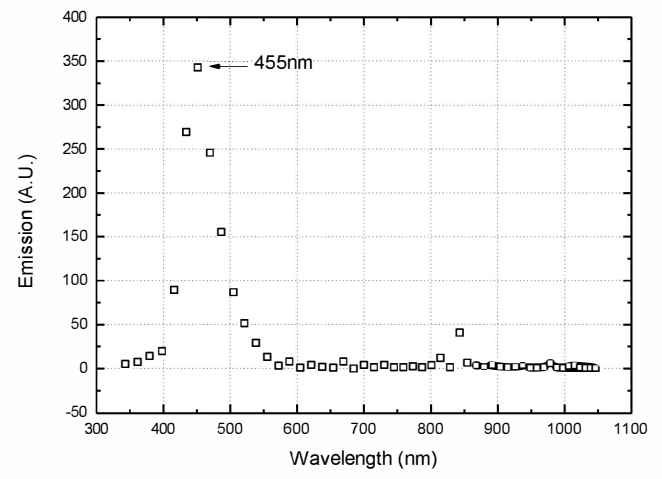

Fig.2. Light emission spectrum of the plastic scintillator under $100 \mathrm{keV} \& 200 \mathrm{~m}$ As X-ray

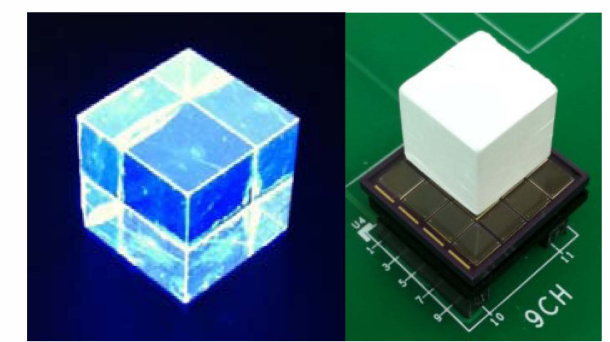

Fig.3. (Left) The plastic scintillator under ultraviolet rays, (Right) The detector module coupled with the SiPM and the plastic scintillator. The plastic scintillator is covered with Teflon tape and it stick on SiPM by the BC-630 optical grease.

\section{Experimental setup}

The electronics for counting the number of radiation interacting with plastic scintillator were consisted of preamp, main amp and Single Channel Analyzer (SCA) on Printed Circuit Board (PCB) designed by KAIST. The amplifiers as preamp, main amp and SCA used the OPA656 commercial OPAMP. The output line from shared anodes of SiPMs was connected to preamp. Then, the preamp was connected to main amp to control the voltage gain by variable resister. The output signal from Low Level Discriminator (LLD) and Upper Level Discriminator (ULD) in SCA as the final outcome, these were counted by two NIM Timer and Counter, No. 871, Ortec. So, the equivalent dose or the equivalent dose rate could be calculated by multiplying the conversion factor to the count rate and total counts. The schematic of electronics is in Fig. 4. This study used the pulse counting dosimetry. If the detector module needs to find the wide energy range of radiation for the other radio isotopes, the detector module will be covered with $2 \mathrm{~mm}$ aluminum filter [10].

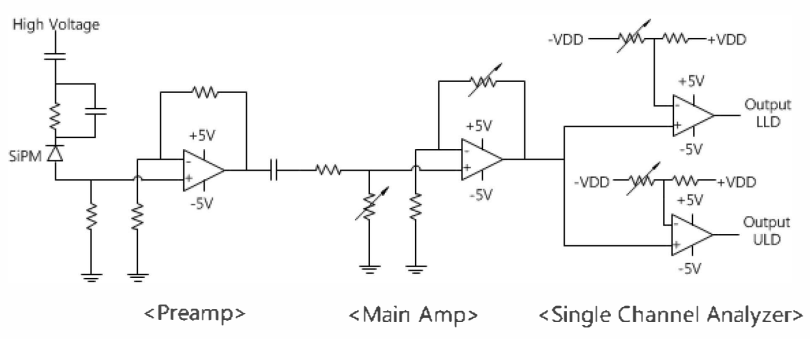

Fig.4. Schematic of electronics designed in KAIST

All experiments used the reference radiation irradiation facility in Korea Hydro \& Nuclear Power Co., LTD (KHNP) central research institute in Republic of Korea. The all of irradiation facilities in KHNP are certified by Korea Laboratory Accreditation Scheme (KOLAS). The radio isotope for experiment was Cs-137. Reference irradiation dose for equivalent dose rate was from $1 \mathrm{uSv} / \mathrm{h}$ to $900 \mathrm{mSv} / \mathrm{h}$, and, for equivalent dose was from $1 \mathrm{uSv}$ to $1 \mathrm{mSv}$.

\section{RESULTS}

The experiment result for dose rate linearity is in Fig.5. Irradiated equivalent dose rate was from $1 \mathrm{uSv} / \mathrm{h}$ to $900 \mathrm{mSv} / \mathrm{h}$. From this result, the dose rate starts to saturate over the $100 \mathrm{mSv} / \mathrm{h}$ condition, so the maximum countable dose rate was $18 \mathrm{mSv} / \mathrm{h}$. MCNP result under same condition is almost similar with experiment result. But, the saturation dose not happened over $18 \mathrm{mSv} / \mathrm{h}$ in simulation, because MCNP could not consider decay time of preamp and main amp.

From this experiment result, we can calculate the conversion coefficient between the counts rate and the equivalent dose rate. The calculated dose rate conversion coefficient is in Eq. 1 .

$$
\mathrm{X}[\mathrm{mSv} / \mathrm{h}]=2.79 \times 10^{-4} \times \mathrm{Y}[\text { Counts per Sec. }] \ldots \ldots . . . \text { Eq. } 1
$$

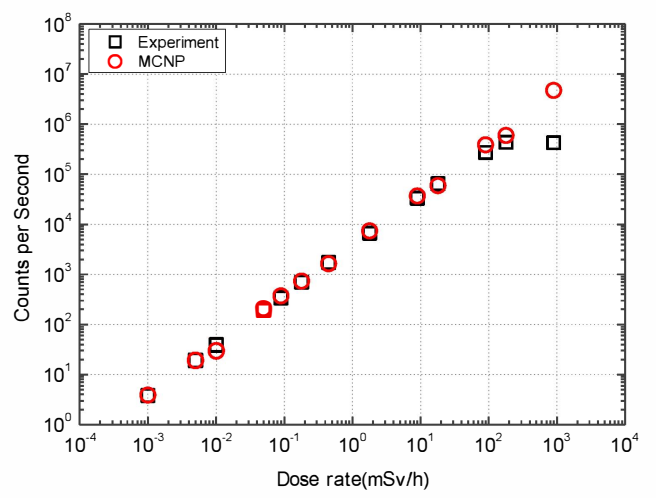

Fig.5. Dose rate linearity (square : experiment, Circle : MCNP)

The experiment result of dose linearity is in Fig.6. Irradiated equivalent dose was from $1 \mathrm{uSv}$ to $1 \mathrm{mSv}$ and the linearity was guaranteed in the whole ranges. The linearity of equivalent 
dose is mainly decided by incident flux as dose rate. So, in this dose range which is not happen the saturation from $1 \mathrm{uSv} / \mathrm{h}$ to $18 \mathrm{mSv} / \mathrm{h}$, the linearity of equivalent dose is kept continuously. MCNP result under same condition is almost similar with experiment result. The calculated dose conversion coefficient is in Eq. 2.

$$
\mathrm{X}[\mathrm{mSv}]=7.74 \times 10^{-8} \times \mathrm{Y}[\text { Counts }]
$$

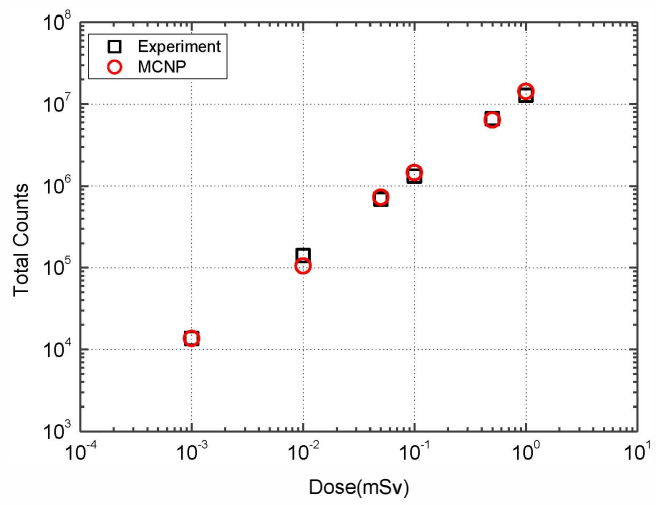

Fig.6. Dose linearity (square : experiment, Circle : MCNP)

\section{CONCLUSION}

In this study, we suggested the radiation detector coupled with the states of arts silicon detector, SiPM, and the plastic scintillator has similar energy absorption coefficient with human tissue, and then, evaluate the availability of detector module for Cs-137 dosimetry.

The summary of experiment results are as follows. First, the dosimetry characteristic is expressed in the count difference depends on equivalent dose rate and equivalent dose, and it is correspond with MCNP calculation. Second, the conversion coefficient between number of counts and radiation dose is calculated, and, it keeps the linearity in the range that equivalent dose rate is from $1 \mathrm{uSv} / \mathrm{h}$ to $18 \mathrm{mSv} / \mathrm{h}$, equivalent dose is from $1 \mathrm{uSv}$ to $1 \mathrm{mSv}$. The linearity depends on radiation flux and the decay time of preamp and main amp.

These experiment results means the SiPM with the plastic scintillator is able to use the dosimetry application for Cs-137 dosimetry. New detector module has the advantages that smaller volume than GM Tube or ion chamber and the lower price than inorganic scintillator as $\operatorname{CsI}(\mathrm{Tl})$. So, it will helpful to relieving the public anxiety about radiation exposure by popularizing the dosimetry device.

\section{REFERENCES}

[1] B. Dolgoshein, et al., Nucl. Instr. And Meth. A 563(2006)368.

[2] W.W. Moses, Nucl. Instr. And Meth. A 580(2007) 919

[3] J. Y. Park, et al., Sae-Mulli 52,(R) 390(2006)
[4] A. Nassalski, et al., IEEE Trans. On Nucl. Sci., Vol. 55 No. 3 (2008)

[5] J.H. Hubbel, Int. J. Appl. Radiat. Isot., Vol.33, pp. 1269 to 1290 (1982)

[6] Johnson, E. B., et al., In Aerospace Conference, 2010 IEEE (pp. 1-8). IEEE. (2010)

[7] Glenn F. Knoll, Radiation detection and measurement (fourth edition), Wiley

[8] A. Kuhn, et al., IEEE Trans. Nucl. Sci. NS-51 (2004) 2550

[9] W.W. Moses, et al., IEEE Trans. Nucl. Sci. NS-46 (1999) 474

[10] Jung, M., Radiation protection dosimetry, 51(3), 157-167. (1994) 